\title{
Body weight distributions of central European Coleoptera
}

\author{
WERNER ULRICH \\ Nicolaus Copernicus University in Toruń, Department of Animal Ecology, Gagarina 9, 87-100 Toruń; Poland; \\ e-mail: ulrichw @ uni.torun.pl
}

Key words. Beetles, body weight, size ratios, speciation

\begin{abstract}
Species number - body weight distributions are generally thought to be skewed to the right. While this pattern is well documented in vertebrates, comparative studies on species rich invertebrate taxa are still scarce. Here I show that the weight distributions of central European Coleoptera (based on 8257 species body weight data compiled from Freude et al., 1964-1994) are predominantly right skewed. Skewness and species richness per taxon were positively correlated. The number of modes of the body weight distributions was negatively correlated with species richness. 273 of the 558 genera had bimodal distributions. Species richness per genus did not significantly depend on mean genus body weight. In general the coleopteran size distributions differed from those of European Hymenoptera but were similar to the respective distributions of vertebrates. I conclude that we should be cautious when generalizing patterns found in one taxon.
\end{abstract}

\section{INTRODUCTION}

The study of animal body sizes has a long tradition in ecology (Peters, 1983; Calder, 1984; Schmidt-Nielsen, 1984; Gotelli \& Graves, 1996). Recently, species size distributions (SSDs) have regained interest after the notion that there are taxon specific differences in SSDs that might be explained by differential patterns of speciation and extinction (Dial \& Marzluff, 1988; Allen et al., 1999; Knouft \& Page, 2003; Ulrich, 2006) and by evolutionary trends towards larger or smaller body sizes (Orme et al., 2002; Smith et al., 2004). Hence, SSDs might be tools to link evolutionary processes to ecological patterns (Etienne \& Olff, 2004; Ulrich, 2005, 2006).

Our current knowledge about the ecological implications of animal body sizes stems mostly from studies of vertebrate taxa (cf. Peters, 1983; Calder, 1984; SchmidtNielsen, 1984; Brown, 1995; Kozłowski \& Gawelczyk, 2002; Smith et al., 2004). From this work five major patterns emerged:

- Classical niche based models (Hutchinson \& MacArthur, 1959; May, 1986) and theoretical work based on fractal geometry (Morse et al., 1985) predict the lower weight classes to be most species rich. The empirical evidence rather points to medium size classes as being most diverse and therefore to unimodal humped distributions (Dial \& Marzluff, 1988; Brown, 1995; Novotný \& Kindlmann, 1996; Kozłowski \& Gawelczyk, 2002; Smith et al., 2004; Ulrich, 2006).

- SSDs (based on log body weight or log body length) appeared to be considerably right skewed with more small than large bodied species (small and large is here always used with respect to the mean body size of a given taxon) (Gaston \& Blackburn, 2000; Kozłowski \& Gawelczyk, 2002; Smith et al., 2004). This pattern is frequently explained in terms of intra- (Kozłowski \& Weiner, 1997) and interspecific (Brown et al., 1993) body size optimization or body size dependent speciation and extinction rates (McKinney, 1990; Maurer et al., 1992).

- Mammal size distributions become more symmetrically distributed at small geographic scales (Bakker \& Kelt, 2000). Such a pattern implies a selective species assembly caused either by an accumulation of larger species at these scales or by a selective loss of smaller species.

- The degree to which SSDs are skewed appears to depend on taxonomic level. Higher levels were found to have a more pronounced skew and therefore a higher proportion of small species (Kozłowski \& Gawelczyk, 2002). This implies a positive correlation of SSD skew and species richness (Ulrich, 2006).

- Body size within vertebrate taxa seems to be phylogenetically constrained. The study of Smith et al. (2004) on constraints on mammalian body size showed that these constraints (measured as the coefficient of correlation of congeneric species pairs) are strongest in medium size classes.

SSDs of terrestrial invertebrates are much less studied (Gunnarson, 1990; Basset \& Kitching, 1991; Novotný \& Kindlmann, 1996; Ulrich, 2005, 2006). Chislenko (1981) published size distributions of all major insect orders and reported for nearly all of them symmetric body size distributions. Recently, Espadaler \& Gomez (2002) and Ulrich (2006) published regional SSDs of Iberian ant species and European Hymenoptera, respectively. Both studies found hymenopteran SSDs to differ from the vertebrate pattern. At all taxomonic levels and irrespective of taxon species richness SSDs appeared to be symmetric. Further, body size is less constrained than in vertebrates (Ulrich, 2006). In turn, irrespective of taxonomic level medium size classes appeared to be most species rich and unimodal SSDs dominate.

Coleopteran body size distributions are currently only poorly known. Chislenko (1981) reported a unimodal 
symmetric SSD (skewness $\gamma=0.08$, n.s.) for the Coleoptera known to him (4875 species). However, this work used a rather heterogeneous ensemble of species from different geographical regions. Novotný \& Kindlmann (1996) analyzed data on parts of the central European Coleoptera (5790 species) contained in Reitter (1908-1916) to assess skewness and the number of modes. They found a weak trend towards right skewed SSDs but concluded that this skew might be significant mainly due to the large number of species analyzed. The present paper aims to examine the body size distributions of the central European Coleoptera as contained in the systematic work of Freude et al. (1964-1994) comprising more than 8700 species. From a comparison with similar work on European Hymenoptera (Ulrich, 2006) and world vertebrates (Smith et al., 2004) it will be shown that taxon specific size distributions exist and that we have to be careful when generalizing based on single taxon patterns.

\section{MATERIAL AND METHODS}

The present study is based on the treatise of central European Coleoptera of Freude et al. (1964-1994). From this work I compiled a database that contains 8727 species from 1685 genera and 112 families (Table 1). For 8257 species body length data are available $(94.6 \%)$. The classification of species above the genus level follows the tree of life project (http://tolweb.org/tree/). The database contains the following taxonomic and morphometric entries: suborder, superfamily, family, subfamily, genus, subgenus, species, minimum, maximum and mean body length, and body weight.

Because most studies use body weight as the basic measure of size (cf. Gaston \& Blackburn, 2000, Kozlowski \& Gawelczk, 2002) the present work is based on mean species dry weight calculated from the arithmetic mean of available data on minimum and maximum body length using the regression equation of Ganihar (1997)

$$
W[\mathrm{mg}]=0.038 L[\mathrm{~mm}]^{2.46}
$$

Of course, in the majority of species the literature-based mean lengths will only be rough estimates. However, these inaccuracies are counterbalanced by the large number of data points used for the analysis. Body weight distributions (in the following the term SSD refers always to the species - body weight distribution) are based on $\ln$-transformed weights. Skewness $\gamma$ is computed as in Ulrich (2006):

$$
\gamma=\frac{\mathrm{n}}{(\mathrm{n}-1)(\mathrm{n}-2)} \sum_{\mathrm{i}=1}^{\mathrm{n}}\left(\frac{\mathrm{w}_{\mathrm{i}}-\overline{\mathrm{w}}}{\sigma_{\mathrm{w}}}\right)^{3}
$$

where $w_{i}$ is the ln-transformed body weight of species i. I calculated the standard error of $\gamma$ according to Tabachnick \& Fidell (1996): $\operatorname{SE}(\gamma)=(6 / \mathrm{n})^{1 / 2}$.

To assess the number of modes of the body weight distributions I used a normal kernel density estimator according to Havlicek \& Carpenter (2001):

$$
f_{h}(x)=\frac{1}{S h \sqrt{2 \pi}} \sum_{i=1}^{S} \operatorname{Exp}\left(-\frac{1}{2}\left(\frac{x_{i}-x}{h}\right)^{2}\right)
$$

with $\mathrm{S}$ being the number of species, $\mathrm{x}_{\mathrm{i}}$ the respective $\ln$ transformed body weights, and $\mathrm{h}$ the band width. I used a smooth bandwidth according to Silvermann (1986) of

$$
h-1.06 S^{-0.2} \min \left(\sigma_{x} ;\right. \text { range/5.36) }
$$

with range being the range of $\ln$ transformed body weights. The step width $\mathrm{x}$ was in all cases $\mathrm{h} / 5$. Kernel density estimates were done for all genera with at least five species.

To study whether within genus body weights are constrained within upper and lower limits (phylogenetic constraint) I fol-
TABLE 1. Basic entries of the coleopteran database. Numbers of families, genera and species included in the analysis of 18 European superfamilies of Coleoptera.

\begin{tabular}{lcccc}
\hline & \multicolumn{4}{c}{ Numbers of } \\
\cline { 2 - 5 } Superfamily & Families & Genera & Species & $\begin{array}{c}\text { Species with } \\
\text { body length data }\end{array}$ \\
\hline Bostrychoidea & 4 & 50 & 155 & 146 \\
Buprestoidea & 1 & 27 & 133 & 129 \\
Byrrhoidea & 1 & 12 & 39 & 39 \\
Cantharoidea & 5 & 19 & 118 & 116 \\
Caraboidea & 7 & 131 & 1002 & 978 \\
Chrysomeloidea & 3 & 182 & 961 & 925 \\
Cleroidea & 3 & 49 & 143 & 135 \\
Cucujoidea & 38 & 334 & 1281 & 1215 \\
Curculionoidea & 9 & 298 & 1482 & 1449 \\
Dascilloidea & 4 & 11 & 44 & 44 \\
Dermestoidea & 4 & 16 & 59 & 59 \\
Dryopoidea & 5 & 18 & 64 & 60 \\
Elateroidea & 4 & 68 & 215 & 201 \\
Histeroidea & 2 & 38 & 112 & 107 \\
Hydrophiloidea & 4 & 25 & 198 & 178 \\
Lymexyloidea & 1 & 2 & 8 & 3 \\
Scarabaeoidea & 4 & 58 & 245 & 241 \\
Staphylinoidea & 13 & 347 & 2468 & 2232 \\
\hline All & 112 & 1685 & 8727 & 8257 \\
\hline
\end{tabular}

lowed Smith et al. (2004) and Ulrich (2006) and computed the regressions of $\ln$ transformed body weights between congeneric species pairs. For genera having two to ten species, all pairs were included; for larger genera, $20 \%$ of all species pairs were randomly selected. The coefficient of correlation $r$ is then a measure of how much body size is constrained within a given taxon (Smith et al., 2004). I tested $r$ against two null model approaches and assigned species body weights within each genus using a normal random distribution around the observed mean according to a proportional rescaling process $\left(\sigma^{2}=\mu^{2}\right)$ and according to a Poisson distribution $\left(\sigma^{2}=\mu\right)$. It should be emphasized that such a comparison is not a true phylogenetic analysis where body size distributions are compared with respect to the underlying phylogenetic tree. Such an analysis is still impossible due to the low phylogenetic resolution of the Coleoptera.

To test whether observed body weights are regularly spaced within the observed ranges, I computed the ratios of logtransformed body weights $\left(\mathrm{R}=\ln \mathrm{W}_{\mathrm{n}} / \ln \mathrm{W}_{\mathrm{n}-1}\right)$ and raw body weights $\left(\mathrm{R}=\mathrm{W}_{\mathrm{n}} / \mathrm{W}_{\mathrm{n}-1}\right)$ of subsequent species on the rank order of species for all genera with more than five species (the ratio test of Strong et al., 1979). I compared the variance of these ratios with the expectation from a null model, where species body lengths were randomly placed inside the respective range (two species were placed at the upper and lower observed length or weight, cf. Gotelli \& Graves, 1996). Respective confidence limits of the null models were obtained from 1000 replicates. To compare taxa I calculated the standardized effect size SES of $R$ as a $Z$-transformed score $[Z=(R-\mu) / \sigma]$ to compare the observed index $R$ to the distribution of simulated indices $(\mu=$ mean and $\sigma$ $=$ standard deviation of the simulated ratios). SES values below -2.0 or above 2.0 indicate approximate statistical significance at the $5 \%$ error level (1-tailed test). 

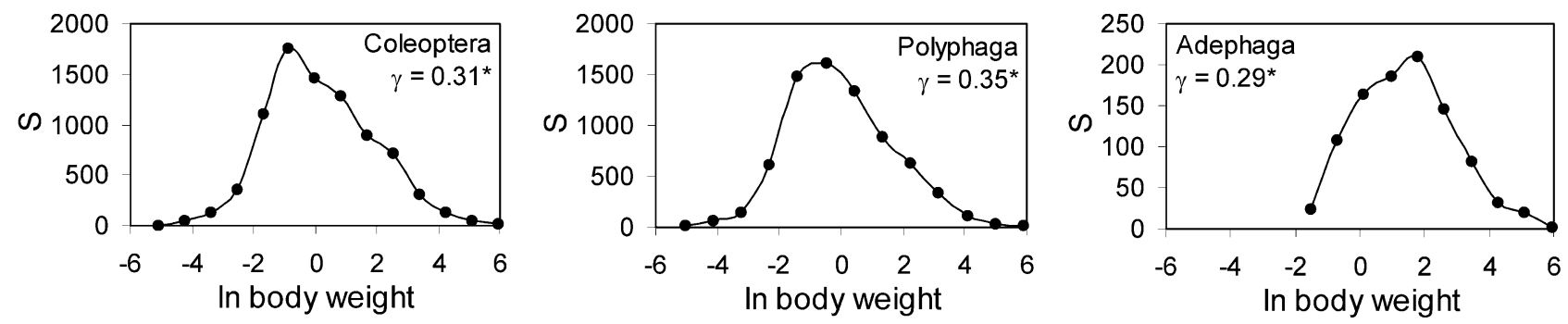

Fig. 1. Size distributions of all central European Coleoptera and the two suborders Polyphaga and Adephaga. Data points denote numbers of species per size class. The number of data points reflects the number of size classes considered. Sizes are given as $1 n$ transformed body weights in $\mathrm{mg}$. An asterisk after the skewness $(\gamma)$ denotes that it is significant at $\mathrm{p}<0.01$.

Regression slopes refer to ordinary least squares regressions computed with the multiple regression and the non-linear estimation modules of Statistica 7 (Statsoft, 2005). Errors are standard errors.

\section{RESULTS}

The central European Coleoptera range from about $0.004 \mathrm{mg}$ dry weight [Scaphisoma (Scaphidiidae) and Oligella (Ptilidae) species] to more than $500 \mathrm{mg}$ dry weight in Procerus gigas (Carabidae) and Lucanus cervus (Lucanidae). They span a body weight range of more than five orders of magnitude.

The body weight distributions of the suborders and all Coleoptera (Fig. 1) were significantly $[p(\gamma=0)<0.01]$ right skewed. Eleven of the 18 superfamily SSDs (Fig. 2) were significantly right, and five significantly left skewed. The Chrysomeloidea were significantly right skewed $(p<0.05)$ and bimodal, a pattern that mainly reflects that it is composed of two families (Chrysomelidae and Cerambycidae).

12 of the 77 family skewness values were significantly positive, five negative (Fig. 3A). Eight of the 18 families with more than 100 species had significantly $(p<0.001)$ right skewed SSDs. Of 403 genera with at least five species 196 had left and 207 right skewed SSDs (Fig. 3B). These values do not differ from the null expectation of a random distribution around zero $\left[\mathrm{p}\left(\chi^{2}\right)>0.1\right] .28$ genera (6.9\%, Fig. 3B) ranged outside the $99 \%$ confidence limits of the skewness.

The above results might indicate a trend towards positive skewness in higher taxa. A Kruskal Wallis ANOVA comparing genera, subfamilies, families, and superfamilies with respect to mean skewness did not point to increasing mean skew with taxonomic level $(\mathrm{P}>0.5)$. However, there was a highly significant positive correlation $(\mathrm{p}<0.001)$ between skewness and $\ln$ species richness per family (Fig. 3A). This correlation remained when genera, subfamilies, and superfamilies were included $\left(\mathrm{R}^{2}\right.$ $=0.06 ; \mathrm{p}<0.001)$. Using the subfamily to order levels only strengthened the correlation to $\mathrm{R}^{2}=0.16$ and $\mathrm{p}<$ 0.0001 (Fig. 4). Of 34 taxa above the genus level with at least 100 species 26 had a positive SSD skewness. All but one taxon with more than 200 species (14 taxa) had a positive skew, 12 of these were statistically significant at the $1 \%$ error level.

In none of the superfamilies (Fig. 2), families and genera (data not shown) with at least five species was the lowermost or the uppermost size class the species richest. Nevertheless the species richness - log mean taxon body weight plots were at the superfamily, family and genus level (Fig. 3D) not significantly unimodal. All quadratic and linear terms of fits of quadratic algebraic functions to the data sets (using ln transformed species numbers) were not significant [all $\mathrm{p}(\mathrm{t})>0.05$ ]. Further, seven genera of the first body weight quartile (mean weight $<0.19 \mathrm{mg}$, in total 51 genera) had more than 20 species, whereas from the overall frequency distribution ten such genera were expected (Fig. 3D). Six genera of the fourth quartile (mean weight $>10 \mathrm{mg}$, in total 51 genera) had more than 20 species although again ten were expected. Both deviations from expectation are statistically not significant (binomial test $\mathrm{P}>0.5$ ). Hence, the unimodal impression of Fig. 3D is not corroborated by the statistical analysis and arises due to a higher number of genera of intermediate size.

Variance - mean ratios of genus body lengths were consistent with a proportional rescaling pattern (Taylor' power law, Taylor, 1960) with a scaling exponent $\mathrm{z}=$ 2.23 (Fig. 3C). The ratio test pointed to clumped distributions in $\ln$ body weight within coleopteran genera. In 84 of the 403 genera with at least five species (20.8\%) the observed variance of the $\ln$ weight ratios $\ln \mathrm{W}_{\mathrm{n}} / \ln \mathrm{W}_{\mathrm{n}-1}$ was above the upper $95 \%$ confidence level of the null model. Using raw body weight ratios $\mathrm{W}_{\mathrm{n}} / \mathrm{W}_{\mathrm{n}-1}$ resulted in only 34 genera $(8.4 \%)$ above the $95 \%$ null model confidence level. Hence, body weight appeared to be randomly distributed with respect to a linear random null model. Further there was a strong positive correlation between effect size $\mathrm{Z}$ and species richness $\left(\mathrm{R}^{2}=0.50 ; \mathrm{P}<\right.$ $0.0001)$. Of 38 genera with more than 30 species 31 had positive Z-values $>1.96$. Their body length distribution was therefore aggregated.

Body size of Coleoptera appeared to be highly constrained when measured by the coefficient of correlation using intragenus pairwise comparisons (Fig. 5A). The coefficient of correlation was $r=0.91$. The two null model approaches in turn gave $r=0.47$ (rescaling, Fig 5B) and $r=0.67$ (Poisson variability, Fig 5C). The observed intragenus variability in body size is therefore much lower than expected from both null models.

Of the 558 genera with at least five species 45 had unimodal, 273 bimodal, and 85 trimodal SSDs. The number of modes was significantly negatively correlated with 

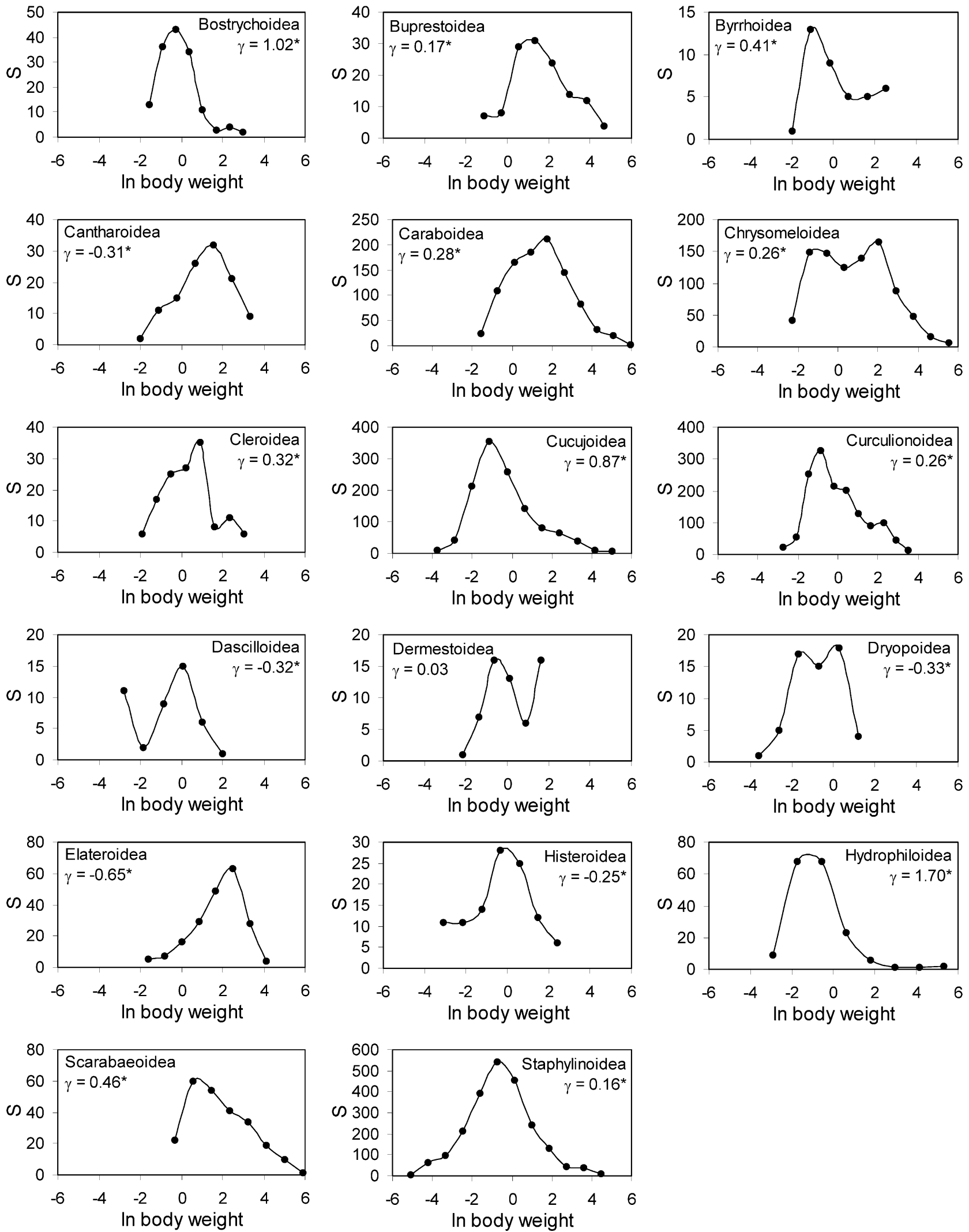

Fig. 2. Size distributions of the superfamilies of central European Coleoptera having more than 30 species. Data points denote numbers of species per size class. The number of data points reflects the number of size classes considered. Sizes are given as $1 n$ transformed body weights in $\mathrm{mg}$. An asterisk after the skewness $(\gamma)$ denotes that it is significant at $\mathrm{p}<0.01$. 

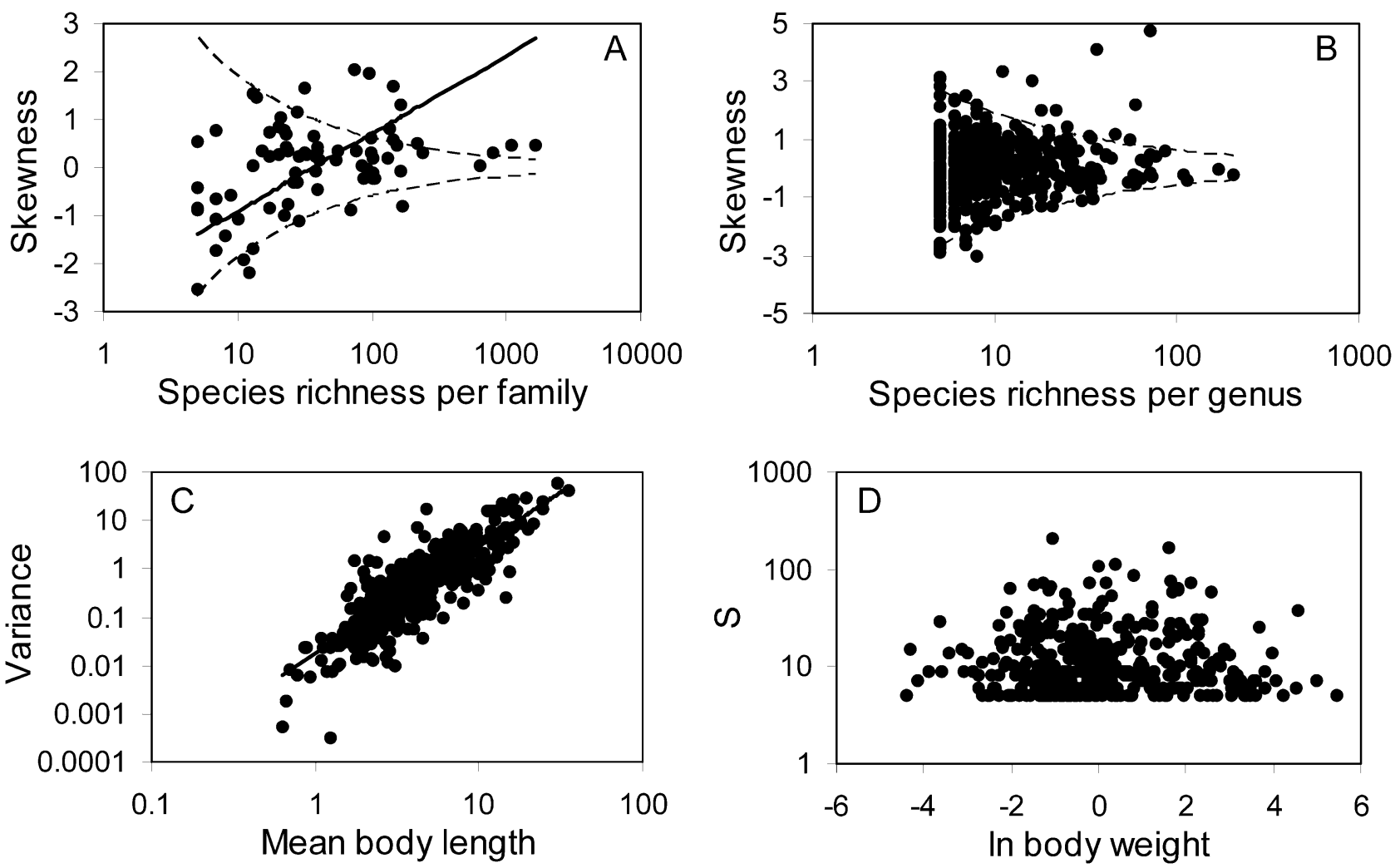

Fig. 3. A, B: Skewness of the body size distributions (ln transformed body weights in $\mathrm{mg}$ ) of coleopteran families (A) and genera (B) for all taxa with at least 5 species (S). Given are also the upper and lower $99 \%$ confidence limits according to the approximation of Tabachnick \& Fidell (1996) (broken lines). The full line in A shows the RMA regression $\gamma=-2.52+0.70 \ln (\mathrm{S}) ; \mathrm{R}^{2}=0.14 ; \mathrm{p}<$ 0.001. C: The variance - mean length relationship $\left(\sigma^{2}=\mu^{z}\right)$ of central European Coleoptera follows closely Taylor's power law with a slope of $z=2.23\left(R^{2}=0.70 ; p<0.0001\right)$ (length is used instead of ln body weight to avoid negative values). D: The species number -ln mean body weight relationship of genera. Included are all genera with more than five species.

species richness $\left(\mathrm{R}^{2}=0.11, \mathrm{P}<0.001\right)$. The respective correlations for subfamilies $\left(\mathrm{R}^{2}=0.21, \mathrm{P}<0.0001\right)$ and families $\left(\mathrm{R}^{2}=0.22, \mathrm{P}<0.0001\right)$ were also highly significant. Both, subfamilies (54\%) and families (53\%) had mostly bimodal SSDs.

\section{DISCUSSION}

Three major findings emerged regarding SSDs in central European Coleoptera: first, coleopteran size distributions are predominantly right skewed; second, size

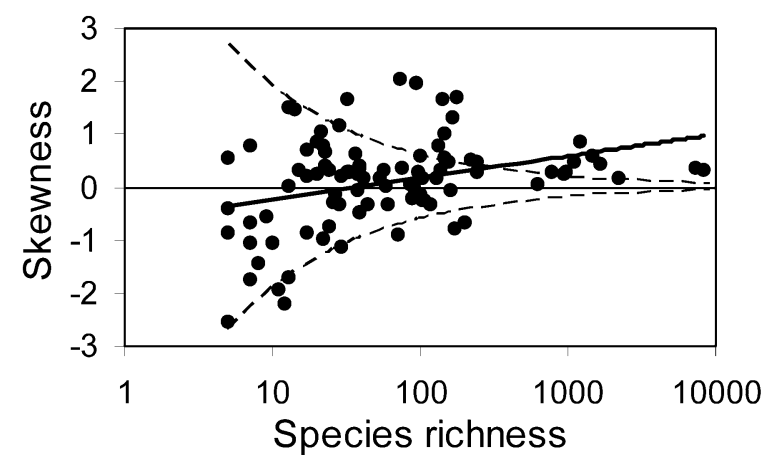

Fig. 4. Skewness values of subfamilies, 112 families, 17 superfamilies, 3 suborders, and all Coleoptera dependent on the species richness of the respective taxonomic level. $\mathrm{R}^{2}=0.12 ; \mathrm{p}$ $<0.0001$. distributions appeared to be taxon specific, which implies that we must be careful when generalizing about possible causes of observed distributions; third, intrataxon variability in body size is less than expected from a random distribution pointing to certain phylogenetic constraints on body size.

The present study also allowed for the first time a detailed comparison of body size distributions of two large European arthropod taxa. In a previous study (Ulrich, 2006) I found hymenopteran SSDs to be predominantly unimodal and symmetrical (whole order $\gamma=$ -0.04). Chislenko (1981) and Espadaler \& Gomez (2002) reported a very similar pattern. The central European Coleoptera of the present study deviate from this pattern. Their size distributions are at least above the genus level predominantly right skewed (Figs 1-3). Novotný \& Kindlmann (1996) have reported already a skew of $\gamma=$ 0.27 ( $\mathrm{P}<0.001)$ for central European Coleoptera, a value very similar to the present one $(\gamma=0.31)$. Further, I found at different taxonomic levels significant positive correlations between skewness and species richness. Such a pattern does not exist in the Hymenoptera (Ulrich, 2006) but resembles the one known from vertebrates (Kozłowski \& Gawelczyk, 2002, Smith et al., 2004).

Many evolutionary and ecological scenarios have been proposed to explain right skewed SSDs (reviews in 

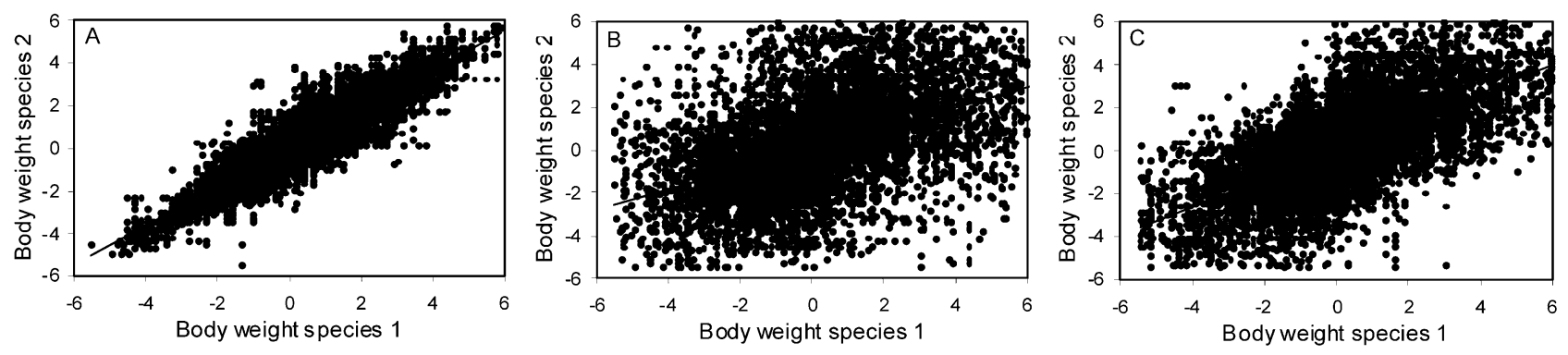

Fig. 5. A: Regression of body weights of congeneric species pairs for all coleopteran genera with at least five species. Phylogenetic constraint (coefficient of correlation) $r=0.91$. B: The same regression for the null model where within genus body weights were randomly assigned with a variance of $\sigma^{2}=\mu^{2}$ (proportional rescaling): $r=0.47$. C: The same regression for the null model having a Poisson distribution of body weights $\left(\sigma^{2}=\mu\right): r=0.67$.

Brown, 1995; Gaston \& Blackburn, 2000; Kozłowski \& Gawelczyk, 2002). McKinney (1990) assumed that body weight evolution can be modelled by a random walk process and termed this the random diffusion model. In such a process upper or lower boundaries of body size (modelled as reflecting boundaries) result in SSD skews to the opposite direction. If the boundary model were to apply, the genera close to these boundaries should exhibit SSD skews in opposite directions. For the Hymenoptera I found size class specific SSDs in accordance with the random diffusion model (Ulrich, 2006). Using a sliding window technique to infer a trend in mean skewness a highly significant $\left(\mathrm{R}^{2}=0.07, \mathrm{p}<0.0001\right)$ negative regression between mean skewness per genus and mean ln body weight (slope $=-0.05 \pm 0.006$ ) appeared. Applying the same technique to Coleoptera resulted in a very different picture (Fig. 6). A significant U-shaped distribution came up with the genera of the lower and upper size classes having mainly positive SSD skews and the middle ranking sizes classes negative skews. This pattern is at least for the upper size classes not in accordance with the diffusion model where symmetrical or left skewed SSDs are predicted.

However, the pattern might be in accordance with the hypothesis that a taxon specific speciation/extinction bias exists (Alroy, 2000). According to the bias model higher speciation rates of smaller species and/or higher extinc-

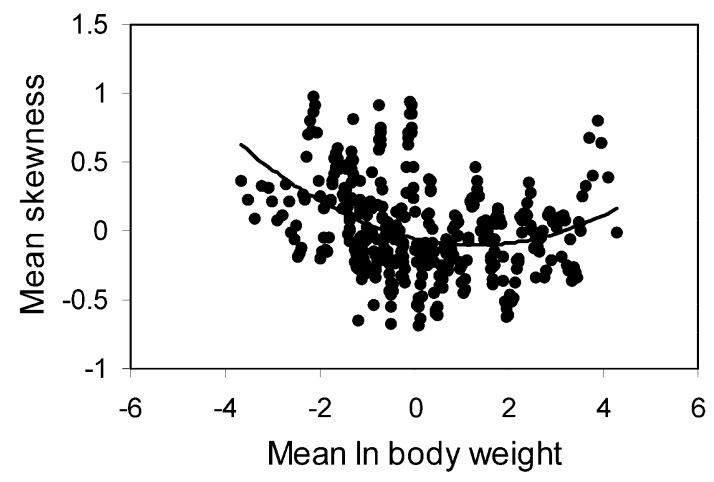

Fig. 6. A plot of mean skewness for a sliding window of 10 genera shifted along the ranked body weights was significantly U-shaped as inferred from the significance level $(p<0.0001)$ of the quadratic term of a second order algebraic function fitted to the data. $\mathrm{R}^{2}=0.13$ tion rates of larger species should result in a right skewed SSD (Knouft \& Page, 2003). If this model were to apply, genera of smaller sized species should be most species rich. This was not the case (Fig. 3D). Species richness did not significantly differ between genera of different mean size. Again this pattern was different from that of the Hymenoptera, but also from that of the Vertebrata, where genera of medium sized species are most species rich (Smith et al., 2004; Ulrich, 2006).

As in the case of Hymenoptera (Ulrich, 2006) and of vertebrates (Smith et al., 2004) body size of Coleoptera appeared to be highly constrained ( $r=0.91$; Fig. 5A). Both null model approaches gave much lower values of $r$ (Figs 5B and C) pointing to a lower variability in body sizes around the genus means than expected from simple statistical distributions. Smith et al. reported $r=0.89$ for vertebrates and I found a very similar value of $r=0.91$ for Hymenoptera. These high values imply that the overlap in size between genera is less than expected from both null models. The remarkable resemblance of $r$ indicates that body sizes of these three very different taxa are constrained in a similar manner and possibly by similar processes.

Smith et al. (2004) argue that the variability of genus or family body sizes should more or less follow a Poisson distribution with a variance / mean ratio of about 1 as indicated by some data of these authors. The variability of hymenopteran body sizes resembled more a proportional rescaling process (Fig. 3F; $\sigma^{2} \propto \mu^{1.81}$ ). The coleopteran pattern (Fig. 3C; $\sigma^{2} \propto \mu^{2.23}$ ) deviates even more from a Poisson distribution indicating that genera of larger bodied species have a proportionally larger span of body weights than smaller bodied genera. Both arthropod patterns are consistent with speciation/extinction processes without distinct upper and lower genus specific boundaries in body size.

In the Hymenoptera within genus variability is higher in species rich genera but independent of body weight (Ulrich, 2006). Hence, body weight distributions in species rich hymenopteran genera appear to be more clumped than expected from the null model. In the Coleoptera I found the same pattern. The Z-tranformed $R$-values of the ratio test were significantly positively correlated with species richness. Both results are consistent with the existence of subgenera that serve as new 
centres of body size (Ulrich, 2006). However, within genus body size distributions differed between Coleoptera and Hymenoptera. In the Hymenoptera log body weight distributions do not differ from the expectation of a linear random null model $(5.9 \%$ of genera above the $95 \%$ null model confidence limit) while raw body weight ratios appear to be clumped (185 genera above the 95\% confidence limit) (Ulrich, 2006). In the Coleoptera this pattern was reversed. For raw body weight ratios $8.4 \%$ and for $\log$ body weight ratios $20.8 \%$ of the genera ranged above the $95 \%$ confidence limit.

This difference might have implications for the question about the unit of evolutionary change. The size distributions of the European Hymenoptera did not deviate from the expectation of a linear random assortment of log body weight ( $\approx$ body length) whereas Coleoptera did not deviate when using raw body weight. This result points to taxon specific scales as being the most appropriate for the study of body size distributions. The result indicates that we should be cautious when using evolutionary models to explain these distributions using standard null model approaches (Gotelli \& Graves, 1996).

In summary, the present results point to differences in body size distributions between major animal taxa. Hence generalizations of patterns and models that extrapolate from findings within one taxon should be treated with caution.

ACKNOWLEDGEMENTS. H. Pearson kindly improved my English. This work was in part supported by grants from the Polish Ministry of Science (KBN, 3 P04F 034 22, 2 P04F 039 29).

\section{REFERENCES}

Allen C.R., Forys E.A. \& Holling C.S. 1999: Body mass patterns predict invasions and extinctions in transforming landscapes. Ecosystems 2: 114-121.

Alroy J. 2000: Understanding the dynamics of trends within evolving lineages. Paleobiology 26: 319-329.

BAKKeR V.J. \& Kelt D.A. 2000: Scale-dependent patterns in body size distributions of neotropical mammals. Ecology 81: 3530-3547.

BASSET Y. \& Kitching R.L. 1991: Species number, species abundance, and body length of arboreal arthropods associated with an Australian rainforest tree. Ecol. Entomol. 16: 391-402.

Brown J.H. 1995: Macroecology. Chicago University Press, Chicago, $270 \mathrm{pp}$.

Brown J.H., Marquet P.A. \& TAPer M.L. 1993: Spatial scaling of species composition: body masses of North American land mammals. Am. Nat. 142: 573-584.

CALDER W.A. 1984: Size, Function, and Life History. Harvard University Press, Cambridge, MA, 431 pp.

Chislenko L.L. 1981: Structure of Fauna and Flora in Connection with Organism Size. Moscow University Press, Moscow.

Dial K.P. \& Marzluff J.M. 1988: Are the smallest organisms the most diverse? Ecology 69: 1620-1624.

Espadaler X. \& Gomez C. 2002: The species body-size distribution in Iberian ants is parameter dependent. Vie Milieu 52: 103-107.

Etienne R.S. \& OlfF H. 2004: How dispersal limitation shapes species - body size distributions in local communities. Am. Nat. 163: 69-83.
Freude H., Harde K.W. \& Lohse G.A. 1964-1994: Die Käfer Mitteleuropas. Goecke \& Evers, Krefeld.

GANiHAR S.R. 1997: Biomass estimates of terrestrial arthropods based on body length. J. Biosciences 22: 219-224.

Gaston K.J. \& Blackburn T.M. 2000: Pattern and Process in Macroecology. Blackwell, Oxford, 377 pp.

Gotelli N.J. \& Graves G.R. 1996: Null Models in Ecology. Smithsonian Institution Press, Washington, xvi +368 pp.

GunNarsson B. 1990: Vegetation structure and the abundance and size distribution of spruce-living spiders. J. Anim. Ecol. 59: 743-752.

HaVliceK T.D. \& CARPENTER S.R. 2001: Pelagic species size distributions in lakes: are they discontinuous? Limnol. Oceanogr. 46: 1021-1033.

Hutchinson G.E. \& MAcArThuR R.H. 1959: A theoretical ecological model of size distributions among species of animals. Am. Nat. 93: 117-125.

Knouft J.H. \& Page L.M. 2003: The evolution of body size in extant groups of North American freshwater fishes: speciation, size distributions, and Cope's rule. Am. Nat. 161: 413-421.

KozŁowsKi J. \& GawelczYK A.T. 2002: Why are species' body size distributions usually skewed to the right? Funct. Ecol. 16: 419-432.

KozłowsKi J. \& WeINER J. 1997: Interspecific allometries are byproducts of body size optimization. Am. Nat. 149: 352-380.

Maurer B.A., Brown J.H. \& Rusler R.D. 1992: The micro and macro in body size evolution. Evolution 46: 939-953.

MAY R.M. 1986: The search for patterns in the balance of nature: advances and retreats. Ecology 67: 1115-1126.

McKinney M.L. 1990. Trends in body size evolution. In McNamara K.C. (ed.): Evolutionary Trends. University of Arizona Press, Tucson, pp. 75-118.

Morse D.R., Lawton J.H., Dodson M.M. \& Williamson M.H. 1985: Fractal dimension of vegetation and the distribution of arthropod body lengths. Nature 314: 731-733.

NovotnÝ V. \& KindLMann P. 1996: Distribution of body sizes in arthropod taxa and communities. Oikos 75: 75-82.

Orme C.D.L., Quicke D.L.J., CoOK J.M. \& Purvis A. 2002: Body size does not predict species richness among the metazoan. J. Evol. Biol. 15: 235-247.

Peters R.H. 1983: The Ecological Implications of Body Size. Cambridge University Press, Cambridge, xii +329 pp.

Reitter E. 1908-1916: Die Käfer des Deutschen Reiches. Lutz, Stuttgart.

Schmidt-Nielsen K. 1984: Scaling: Why is Animal Size so Important? Cambridge University Press, Cambridge, $241 \mathrm{pp}$.

Silvermann B.W. 1986: Density Estimation for Statistics and Data Analysis. Chapman \& Hall, New York, 175 pp.

Smith F.A., Brown J.H., Haskell J.P., Lyons S.K., Alroy J., Charnov E.L., Dayan T., Enquist B.J., ERnest S.K.M., Hadly E.A., Jones K.E., Kaufman D.M., Marquet P.A., Maurer B.A., Niklas K.J., Porter W.P., Tiffney B. \& Willig M.R. 2004: Similarity of mammalian body size across the taxonomic hierarchy and across space and time. Am. Nat. 163: 672-691.

StatSoft Inc. 2005: STATISTICA. Data Analysis Software System), version 7.1. - www.statsoft.com.

Strong D.R., Szyska L.A. \& Simberloff D.S. 1979: Tests of community wide character displacement against null hypotheses. Evolution 33: 897-913.

TABAChNicK B.G. \& Fidell L.S. 1996: Using Multivariate Statistics. $3 r d$ ed. Harper Collins, New York, 880 pp.

TAYLOR L.R. 1960: Aggregation, variance and the mean. Nature 332: 721-722. 
UlRICH W. 2005: Die Hymenopteren einer Wiese auf Kalkgestein: Ökologische Muster einer lokalen Tiergemeinschaft. Berichte des Forschungszentrums Waldökosysteme A 195, $203 \mathrm{pp}$.
UlRICH W. 2006: Body size distribution of European Hymenoptera. Oikos 114: 518-528.

Received October 18, 2006; revised and accepted April 11, 2007 